\title{
Esófago de Barrett: de la metaplasia al cáncer
}

\author{
Miguel Ángel Herrera-Servin* \\ Unidad de Endoscopia Gastrointestinal, Instituto Nacional de Cancerología, Ciudad de México, México
}

\begin{abstract}
Resumen
El esófago de Barrett (EB), condición premaligna relaciona da al desarrollo de adenocarcinoma de esófago, en su diagnóstico se requiere la realización de endoscopia más toma de biopsia y confirmación histológica por dos patólogos expertos, permitiendo un manejo en base a guías. Pero hay aspectos como quienes requieren más atención, la utilidad de las nuevas terapias, diagnóstico de recurrencia. que generan controversias.
\end{abstract}

Palabras clave: Esófago de Barrett. Metaplasia intestinal. Displasia de bajo grado. Displasia de alto grado. Adenocarcinoma de esófago. Recurrencia.

\section{Introducción}

El esófago de Barrett (EB), condición premaligna con una incidencia anual de $0.68 \%$ para progresar a DAG y/o adenocarcinoma ${ }^{1}$, su diagnóstico temprano permite ofrecer el mejor tratamiento en base a los hallazgos endoscópicos e histológicos, pero dentro de ese proceso hay varios puntos que generan dudas o hacen necesario implementar otras estrategias de manejo, puntos que se abordaron en la DDW19 y que se resumirán a continuación.

El diagnóstico de EB indefinido para displasia requiere continuar con tratamiento con IBP a dosis altas y volver a repetir el estudio endoscópico para nueva toma de biopsias, la relevancia de esto se encuentra en revisión sistemática y meta-análisis que realizo AJ. Henn, et al., el cual incluyó 9 estudios con 1,378 pacientes con diagnóstico de EB-IND, donde se reportó una incidencia anual de DAG/ACE y DBG/DAG/ACE en pacientes con EB-IND de $1.3 \%$ y $3.8 \%$ respectivamente, concluyendo que la progresión es mayor que en pacientes sin displasia, teniendo 2 veces más riesgo ${ }^{1}$. Otro aspecto importante es sobre la utilidad de la vigilancia estrecha en EB con DBG, en el trabajo presentado por CS. Dasari, et al., el cual es un estudio prospectivo multicéntrico donde siguieron a 4713 pacientes con EB y displasia de bajo grado, se vio que durante el primer año 4 pacientes progresaron a ACE (incidencia de 1.1/100 pacientes año; 95\%IC: 0.4-2.5), y 20 pacientes progresaron a DAG/ACE (incidencia de 5.4/100 pacientes año; 95\% IC 4.0-8.9). En comparación de aquellos que progresaron después del primer año donde 8 pacientes progresaron a ACE (incidencia de 0.34/100 pacientes año; 95\%IC: 0.2-0.65) y 44 progresaron a DAG/ ACE (incidencia de 1.86/100 pacientes año; 95\%IC: $1.1-2.5)^{2}$. Esto confirma la importancia de la vigilancia de estos pacientes durante el primer año. En cuanto a biomarcadores se mencionó que la expresión del biomarcador KI-67 es útil para predecir progresión a displasia de alto grado y adenocarcinoma de esófago en pacientes con EB y DBG. Se incluyeron 60 pacientes a los que se les realizo inmunohistoquímica con Ki-67 de estos 34 con DBG (57\%) fueron positivos para $\mathrm{KI}-67$, y

\section{Correspondencia:}


de estos, 10 (29.4\%) progresaron a DAG/ACE, los 26 restantes (43\%) negativos para Ki-67 solo 1 progreso a DAG ( $p=.03$, OR $10.42 ; 95 \%$ IC 1.24-87.71), aunque se necesitan más estudios, podría ser de utilidad para establecer una vigilancia más estrecha a aquellos pacientes que expresen este biomarcador ${ }^{3}$.

\section{Tratamiento}

Sobre tratamiento lo más comentado fue la crioablación con balón y la crio ablación con Nitrógeno Líquido. Para valorar la seguridad y eficacia de la ablación multifocal para el tratamiento de erradicación de neoplasia de Barret anteriormente tratada se presentaron los resultados preliminares de un trabajo multicéntrico en EU con 120 pacientes con reporte histopatológico de DBG, DAG y/o CalM, los pacientes fueron tratados mediante crioablación con Balón (1ra Gen. CbFAS Pentax Redwood City, CA) de los 120 pacientes, 19\% tenían ImCa, 53\% DAG y $23 \%$ DBG, $45 \%$ de los pacientes tenían REM previa, se requirieron una media de 2 sesiones (rango 1-3), el éxito técnico se logró en el $96 \%$ de los procedimientos. la CE-D y CE-MI se logró en $100 \%$ y $91 \%$ respectivamente en seguimiento a 12 meses, se presentaron eventos adversos serios en 3 pacientes $(2.5 \%)$, la media de dolor fue de 2/10 (IQR 0-3), 3\% requirieron analgésicos narcóticos, estenosis se presentaron en 16/120 (13\%), la mitad dentro de los 30 días. Concluyendo que este procedimiento tiene alta eficacia, seguro y es tolerable ${ }^{4}$.

En un estudio multicéntrico prospectivo se valoró la eficacia y seguridad del tratamiento de EB mediante crioablación con Nitrógeno Líquido el cual incluyo a 114 pacientes, de los cuales 53\% tenían DAG, $24 \%$ adenocarcinoma intramucoso, DBG/IND 24\% y EBSD $3 \%$, se necesitaron promedio de 3 sesiones (rango 1-4) teniendo como resultado que en el $65 \%$ se logró una CE-MI, y en el $87 \%$ de los casos se logró CE-D, como complicación más importante fue estenosis la cual se presento en un $3.5 \%$. Se confirmo también que la extensión de segmento de Barrett e historia de ablación previa se relacionaban con erradicación incompleta ${ }^{5}$.

\section{Respuesta incompleta, recurrencia y seguimiento}

La resección endoscópica de la mucosa y la Ablación por radiofrecuencia son las dos terapias de elección para el tratamiento de EB asociado a neoplasia, el número de sesiones para una completa erradicación es de 3 , pero hay un grupo de pacientes que requieren más sesiones. En un estudio multicéntrico, prospectivo
(Consorcio TREAT-BE) que incluyo a 583 pacientes, se realizó un análisis multivariado para ver qué factores influyeron en la necesidad de más de 3 sesiones de TEE, se demostró que la presencia de hernia hiatal grande (OR 3.7, 95\% IC 1.1-13.1) y la extensión del segmento de EB (OR 1.4, 95\% IC 1.2-1.7) son predictores significativos de respuesta inadecuada a TEE, por lo tanto pacientes con respuesta incompleta tienen más riesgo de recurrencia de MI (OR 6.1, 95\% IC 1.8-20.2). por lo que en este tipo de pacientes deben plantearse diferentes alternativas de tratamiento y vigilancia ${ }^{6}$. Con respecto a los factores relacionados recurrencia en un metaanálisis presentado por R. Krishnamoorthi, et al., de 41 estudios con 4443 pacientes $^{7}$, y otro estudio realizado por Cotton, et al., con 3478 pacientes del registro de RF de US y del registro Nacional HALO de UK, concluyeron que los mayores predictores relacionados a recurrencia están la edad, la extensión del segmento de Barrett e histología previa como displasia de bajo 0 alto grado. Esta a su vez se presenta principalmente en el primer año como lo demuestra un metaanálisis de Sawas, et al. ${ }^{9}$ que incluyo 22 estudios con 1973 pacientes con completa remisión de la metaplasia intestinal donde se encontró que la incidencia de recurrencia en el primer año para Ml es del 12\% (RR=1.8), Displasia $3 \%(R R=1.9)$ y para DAG/ACE $1 \%$ (RR NS), por lo que la vigilancia debe ser más estricta en el primer año, para evitar el riesgo de lesiones perdidas.

Entre los lugares donde se debe tener mayor atención a la hora de realizar la endoscopía de seguimiento es en la UEG como lo demuestra un estudio multicéntrico internacional que incluyo 3 hospitales de US y 2 de UK con un total de 594 pacientes con RCMI se observó que las recurrencias se presentaron en la UEG en un $76 \%$, contra el $24 \%$ que fueron en esófago. De las que se presentaron en la UEG solo el $14 \%$ fueron visibles, el resto $76 \%$ fueron no displásicas, no visibles, $40 \%$ fue recurrencia de displasia no visible, con respecto a las recurrencias en la parte tubular del esófago el $82 \%$ fueron visibles, el $84 \%$ estuvieron dentro de los $5 \mathrm{~cm}$ de la UEG y solo 5 fueron sub-escamosas ${ }^{10}$.

Con respecto a las recurrencias estas pueden ser tratadas en más del 90\% vía endoscópica, para lesiones nodulares resección endoscópica y para lesiones planas con displasia ablación. Tratar las recurrencias como lesiones primarias.

\section{Conclusiones}

La completa remisión de la MI debe ser el objetivo de la terapia endoscópica, prestar especial atención 
en el primer año tanto a DBG y EB-ID (10\% puede albergar displasia), los biomarcadores identifican a pacientes de riesgo, la ARF es el estándar de manejo, las nuevas técnicas prometen resultados satisfactorios, se necesitan más estudios, prestar atención en el seguimiento a pacientes con Hernia hiatal, EB segmento largo, $>60$ años y revisar a detalle la UEG, todo esto con el fin de detectar pacientes con tratamiento incompleto y recurrencia de enfermedad.

\section{Conflicto de intereses}

No existen conflictos de interés. Sin patrocinio de la industria.

\section{Bibliografía}

1. Sa1139: AJ. Henn, CG.Moscoso, BJ Hanson et al. Risk of dysplastic progression in patients with Barrett's esophagus indefinite for dysplasia: a systematic review and meta-analysis. Poster DDW 2019.
2. Sa1148: CS. Dasari, VT. Chandrasekar, P. Sharma et al. The majority of patients with confirmed LGD in barrett's esophagus progress within the first year of diagnosis: results from a large multicenter be consortium. Poster DDW2019.

3. Sa1143: U.Hayat, H.Mesa, J.Peltola et al. Surface ki-67 expression on histopathology predicts progression to high grade dysplasia and esophageal adenocarcinoma in patients with barrett's esophagus and low grade dysplasia. Poster DDW 2019.

4. Presentación ASGE 77: Canton MI, et al. Safety and efficacy of multifocal cryoballoon ablation for eradication of previously untreated barrett's neoplasia: preliminary results of a large multicenter american trial. DDW2019.

5. Sa1137:S. Eluri, V. Kaul NJ. Shaheen et al. liquid nitrogen spray cryotherapy eradicates dysplasia in $87 \%$ and intestinal metaplasia in $65 \%$ of patients with barrett's esophagus: results of a u.s. multicenter registry. DDW19.

6. 642: C. Mittal, VR. Muthusamay, S. Komanduri et al. Predictors of incomplete response to endoscopic eradication therapy: results from the treatment with resection and endoscopic ablation techniques for Barrett's esophagus (treat-be) consortium, Trabajo oral DDW 2019.

7. R. Krishnamoorthi, S. Singh, K. Ragunathan et al. Risk of recurrence of Barrett's esophagus after successful endoscopic therapy Gastrointest Endosc. 2016 June; 83(6): 1090-1106.e3. DDW 2019 Sp.

8. CC. Cotton, R. Haidry, AP. Thrift et al. Development of Evidence-Based Surveillance Intervals After Radiofrequency Ablation of Barrett's Esophagus Gastroenterology 2018;155:316-326.

9. Sawas T, Iyer PG, Alsawas M. et al. Higher Rate of Barrett's Detection in the First Year After Successful Endoscopic Therapy: Meta-analysis. AM J Gastro 2018. AGA Sp 98-101, Questions and controversias in Barrett's Esophagus. DDW19.

10. Sp101: Prasad G. Iyer. Recurrences and Follow up after Therapy, DDW AGA Symposium. 